\title{
A Note on Approximating Curve with 1-Norm Regularization Method for the Split Feasibility Problem
}

\author{
Songnian $\mathrm{He}^{1,2}$ and Wenlong $\mathrm{Zhu}^{1,2}$ \\ ${ }^{1}$ College of Science, Civil Aviation University of China, Tianjin 300300, China \\ ${ }^{2}$ Tianjin Key Laboratory for Advanced Signal Processing, Civil Aviation University of China, \\ Tianjin 300300, China \\ Correspondence should be addressed to Songnian He, hesongnian2003@yahoo.com.cn
}

Received 21 March 2012; Accepted 6 June 2012

Academic Editor: Hong-Kun $\mathrm{Xu}$

Copyright (C) 2012 S. He and W. Zhu. This is an open access article distributed under the Creative Commons Attribution License, which permits unrestricted use, distribution, and reproduction in any medium, provided the original work is properly cited.

Inspired by the very recent results of Wang and Xu (2010), we study properties of the approximating curve with 1-norm regularization method for the split feasibility problem (SFP). The concept of the minimum-norm solution set of SFP in the sense of 1-norm is proposed, and the relationship between the approximating curve and the minimum-norm solution set is obtained.

\section{Introduction}

Let $C$ and $Q$ be nonempty closed convex subsets of real Hilbert spaces $H_{1}$ and $H_{2}$, respectively. The problem under consideration in this paper is formulated as finding a point $x$ satisfying the property:

$$
x \in C, \quad A x \in Q,
$$

where $A: H_{1} \rightarrow H_{2}$ is a bounded linear operator. Problem (1.1), referred to by Censor and Elfving [1] as the split feasibility problem (SFP), attracts many authors' attention due to its application in signal processing [1]. Various algorithms have been invented to solve it (see [2-13] and references therein).

Using the idea of Tikhonov's regularization, Wang and $\mathrm{Xu}$ [14] studied the properties of the approximating curve for the SFP. They gave the concept of the minimum-norm solution of the SFP (1.1) and proved that the approximating curve converges strongly 
to the minimum-norm solution of the SFP (1.1). Together with some properties of this approximating curve, they introduced a modification of Byrne's CQ algorithm [2] so that strong convergence is guaranteed and its limit is the minimum-norm solution of SFP (1.1).

In the practical application, $H_{1}$ and $H_{2}$ are often $\mathbb{R}^{N}$ and $\mathbb{R}^{M}$, respectively. Moreover, scientists and engineers are more willing to use 1-norm regularization method in the calculation process (see, e.g., [15-18]). Inspired by the above results of Wang and $\mathrm{Xu}$ [14], we study properties of the approximating curve with 1-norm regularization method. We also define the concept of the minimum-norm solution set of SFP (1.1) in the sense of 1-norm. The relationship between the approximating curve and the minimum-norm solution set is obtained.

\section{Preliminaries}

Let $X$ be a normed linear space with norm $\|\cdot\|$, and let $X^{*}$ be the dual space of $X$. We use the notation $\langle x, f\rangle$ to denote the value of $f \in X^{*}$ at $x \in X$. In particular, if $X$ is a Hilbert space, we will denote it by $H$, and $\langle\cdot, \cdot\rangle$ and $\|\cdot\|$ are the inner product and its induced norm, respectively.

We recall some definitions and facts that are needed in our study.

Let $P_{C}$ denote the projection from $H$ onto a nonempty closed convex subset $C$ of $H$; that is,

$$
P_{C} x=\arg \min _{y \in C}\|x-y\|, \quad x \in H .
$$

It is well known that $P_{C} x$ is characterized by the inequality

$$
\left\langle x-P_{C} x, y-P_{C} x\right\rangle \leq 0, \quad \forall y \in C .
$$

Definition 2.1. Let $\varphi: X \rightarrow \mathbb{R} \cup\{+\infty\}$ be a convex functional, $x_{0} \in \operatorname{dom}(\varphi)=\{x \in X: \varphi(x)<$ $+\infty$ \}. Set

$$
\partial \varphi\left(x_{0}\right)=\left\{\xi \in X^{*}: \varphi(x) \geq \varphi\left(x_{0}\right)+\left\langle x-x_{0}, \xi\right\rangle, \forall x \in X\right\}
$$

If $\partial \varphi\left(x_{0}\right) \neq \emptyset, \varphi$ is said to be subdifferentiable at $x_{0}$ and $\partial \varphi\left(x_{0}\right)$ is called the subdifferential of $\varphi$ at $x_{0}$. For any $\xi \in \partial \varphi\left(x_{0}\right)$, we say $\xi$ is a subgradient of $\varphi$ at $x_{0}$.

Lemma 2.2. There holds the following property:

$$
\partial(\|x\|)= \begin{cases}\left\{x^{*} \in X^{*}:\left\|x^{*}\right\|=1,\left\langle x, x^{*}\right\rangle=\|x\|\right\}, & x \neq 0, \\ \left\{x^{*} \in X^{*}:\left\|x^{*}\right\| \leq 1\right\}, & x=0\end{cases}
$$

where $\partial(\|x\|)$ denotes the subdifferential of the functional $\|x\|$ at $x \in X$.

Proof. The process of the proof will be divided into two parts. 
Case 1 . In the case of $x=0$, for any $x^{*} \in X^{*}$ such that $\left\|x^{*}\right\| \leq 1$ and any $y \in X$, there holds the inequality

$$
\|y\| \geq\left\langle y, x^{*}\right\rangle=\|x\|+\left\langle y-x, x^{*}\right\rangle,
$$

so we have $x^{*} \in \partial(\|x\|)$, and thus,

$$
\left\{x^{*} \in X^{*}:\left\|x^{*}\right\| \leq 1\right\} \subset \partial(\|x\|) .
$$

Conversely, for any $x^{*} \in \partial(\|x\|)$, we have from the definition of subdifferential that

$$
\begin{gathered}
\|y\| \geq\|x\|+\left\langle y-x, x^{*}\right\rangle=\left\langle y, x^{*}\right\rangle, \quad \forall y \in X, \\
\|y\|=\|-y\| \geq\left\langle-y, x^{*}\right\rangle=-\left\langle y, x^{*}\right\rangle .
\end{gathered}
$$

Consequently,

$$
\left|\left\langle y, x^{*}\right\rangle\right| \leq\|y\|, \quad \forall y \in X
$$

and this implies that $\left\|x^{*}\right\| \leq 1$. Thus, we have verified that

$$
\partial(\|x\|) \subset\left\{x^{*} \in X^{*}:\left\|x^{*}\right\| \leq 1\right\} .
$$

Combining (2.6) and (2.9), we immediately obtain

$$
\partial(\|x\|)=\left\{x^{*} \in X^{*}:\left\|x^{*}\right\| \leq 1\right\} .
$$

Case 2. If $x \neq 0$, for any $x^{*} \in\left\{x^{*} \in X^{*}:\left\|x^{*}\right\|=1,\left\langle x, x^{*}\right\rangle=\|x\|\right\}$, we obviously have

$$
\left\langle y-x, x^{*}\right\rangle=\left\langle y, x^{*}\right\rangle-\|x\| \leq\|y\|-\|x\|, \quad \forall y \in X,
$$

which means that $x^{*} \in \partial(\|x\|)$, and thus,

$$
\left\{x^{*} \in X^{*}:\left\|x^{*}\right\|=1,\left\langle x, x^{*}\right\rangle=\|x\|\right\} \subset \partial(\|x\|) .
$$

Conversely, if $x^{*} \in \partial(\|x\|)$, we have

$$
\left\langle-x, x^{*}\right\rangle \leq 0-\|x\|=-\|x\|, \quad\left\langle x, x^{*}\right\rangle \leq 2\|x\|-\|x\|=\|x\| ;
$$

hence,

$$
\left\langle x, x^{*}\right\rangle=\|x\| .
$$


On the other hand, using (2.14), we get

$$
\|y\| \geq\|x\|+\left\langle y-x, x^{*}\right\rangle=\|x\|+\left\langle y, x^{*}\right\rangle-\left\langle x, x^{*}\right\rangle=\left\langle y, x^{*}\right\rangle, \quad \forall y \in X,
$$

and consequently,

$$
\begin{aligned}
\|y\| & =\|-y\| \geq\|x\|+\left\langle-y-x, x^{*}\right\rangle \\
& =\|x\|-\left\langle y, x^{*}\right\rangle-\left\langle x, x^{*}\right\rangle \\
& =-\left\langle y, x^{*}\right\rangle ;
\end{aligned}
$$

that is,

$$
-\|y\| \leq\left\langle y, x^{*}\right\rangle
$$

Equation (2.17) together with (2.15) implies that

$$
\left|\left\langle y, x^{*}\right\rangle\right| \leq\|y\|, \quad \forall y \in X
$$

hence, $\left\|x^{*}\right\| \leq 1$. Note that (2.14) implies that $\left\|x^{*}\right\| \geq\left\langle x, x^{*}\right\rangle /\|x\|=1$; we assert that

$$
\left\|x^{*}\right\|=1 \text {. }
$$

Thus we have from (2.14) and (2.19) that

$$
\left\{x^{*} \in X^{*}:\left\|x^{*}\right\|=1,\left\langle x, x^{*}\right\rangle=\|x\|\right\} \supset \partial(\|x\|) .
$$

The proof is finished by combining (2.12) and (2.20).

$\|\cdot\|_{\infty}$ and $\|\cdot\|_{1}$ will stand for $\infty$-norm and 1-norm of any Euclidean space; respectively, that is, for any $x=\left(x_{1}, x_{2}, \ldots, x_{l}\right) \in \mathbb{R}^{l}$, we have

$$
\|x\|_{\infty}=\max _{1 \leq j \leq l}\left|x_{j}\right|, \quad\|x\|_{1}=\sum_{j=1}^{l}\left|x_{j}\right| .
$$

Corollary 2.3. In l-dimensional Euclidean space $\mathbb{R}^{l}$, there holds the following result:

$$
\begin{aligned}
\partial\left(\|x\|_{1}\right) & = \begin{cases}\left\{\xi \in \mathbb{R}^{l}:\|\xi\|_{\infty}=1,\langle x, \xi\rangle=\|x\|_{1}\right\}, & x \neq 0, \\
\left\{\xi \in \mathbb{R}^{l}:\|\xi\|_{\infty} \leq 1\right\}, & x=0,\end{cases} \\
& = \begin{cases}\left\{\xi \in \mathbb{R}^{l}: \xi_{i}=\frac{x_{i}}{\left|x_{i}\right|}, \text { if } x_{i} \neq 0 ; \xi_{i} \in[-1,1], \text { if } x_{i}=0\right\}, & x \neq 0, \\
\left\{\xi \in \mathbb{R}^{l}:\|\xi\|_{\infty} \leq 1\right\}, & x=0 .\end{cases}
\end{aligned}
$$


Let $H$ be a Hilbert space and $f: H \rightarrow \mathbb{R}$ a functional. Recall that

(i) fis convex if $f(\lambda x+(1-\lambda) y) \leq \lambda f(x)+(1-\lambda) f(y)$, for all $0<\lambda<1$, for all $x, y \in H$;

(ii) $f$ is strictly convex if $f(\lambda x+(1-\lambda) y)<\lambda f(x)+(1-\lambda) f(y)$, for all $0<\lambda<1$, for all $x, y \in H$ with $x \neq y$;

(iii) $f$ is coercive if $f(x) \rightarrow \infty$ whenever $\|x\| \rightarrow \infty$. See [19] for more details about convex functions.

The following lemma gives the optimality condition for the minimizer of a convex functional over a closed convex subset.

Lemma 2.4 (see [20]). Let $H$ be a Hilbert space and $C$ a nonempty closed convex subset of $H$. Let $f: H \rightarrow \mathbb{R}$ be a convex and subdifferentiable functional. Then $x \in C$ is a solution of the problem

$$
\min _{x \in C} f(x)
$$

if and only if there exists some $\xi \in \partial f(x)$ satisfying the following optimality condition:

$$
\langle\xi, v-x\rangle \geq 0, \quad \forall v \in C
$$

\section{Main Results}

It is well known that SFP (1.1) is equivalent to the minimization problem

$$
\min _{x \in C}\left\|\left(I-P_{Q}\right) A x\right\|^{2} .
$$

Using the idea of Tikhonov's regularization method, Wang and $\mathrm{Xu}$ [14] studied the minimization problem in Hilbert spaces:

$$
\min _{x \in C}\left\|\left(I-P_{Q}\right) A x\right\|^{2}+\alpha\|x\|^{2}
$$

where $\alpha>0$ is the regularization parameter.

In what follows, $H_{1}$ and $H_{2}$ in SFP (1.1) are restricted to $\mathbb{R}^{N}$ and $\mathbb{R}^{M}$, respectively, and $\|\cdot\|$ will stand for the usual 2-norm of any Euclidean space $\mathbb{R}^{l}$; that is, for any $x=$ $\left(x_{1}, x_{2}, \ldots, x_{l}\right) \in \mathbb{R}^{l}$,

$$
\|x\|=\sqrt{x_{1}^{2}+\cdots+x_{l}^{2}}
$$

Inspired by the above work of Wang and $\mathrm{Xu}$, we study properties of the approximating curve with 1-norm regularization scheme for the SFP, that is, the following minimization problem:

$$
\min _{x \in C} \frac{1}{2}\left\|\left(I-P_{Q}\right) A x\right\|^{2}+\alpha\|x\|_{1}
$$


where $\alpha>0$ is the regularization parameter. Let

$$
f_{\alpha}(x)=\frac{1}{2}\left\|\left(I-P_{Q}\right) A x\right\|^{2}+\alpha\|x\|_{1} .
$$

It is easy to see that $f_{\alpha}$ is convex and coercive, so problem (3.4) has at least one solution. However, the solution of problem (3.4) may not be unique since $f_{\alpha}$ is not necessarily strictly convex. Denote by $S_{\alpha}$ the solution set of problem (3.4); thus we can assert that $S_{\alpha}$ is a nonempty closed convex set but may contain more than one element. The following simple example illustrates this fact.

Example 3.1. Let $C=\{(x, y): x+y=1\}, Q=\{(x, y): x+y=1 / 2\}$ and

$$
A=\left(\begin{array}{ll}
\frac{1}{2} & 0 \\
0 & \frac{1}{2}
\end{array}\right) .
$$

Then $A: \mathbb{R}^{2} \rightarrow \mathbb{R}^{2}$ is a bounded linear operator. Obviously, $S_{\alpha}=\{(x, y): x+y=1, x \geq 0, y \geq$ $0\}$ and it contains more than one element.

Proposition 3.2. For any $\alpha>0, x_{\alpha} \in S_{\alpha}$ if and only if there exists some $\xi \in \partial\left(\|x\|_{1}\right)$ satisfying the following inequality:

$$
\left\langle A^{*}\left(I-P_{Q}\right) A x_{\alpha}+\alpha \xi, v-x_{\alpha}\right\rangle \geq 0, \quad \forall v \in C .
$$

Proof. Let

$$
f(x)=\frac{1}{2}\left\|\left(I-P_{Q}\right) A x\right\|^{2}
$$

then

$$
f_{\alpha}(x)=f(x)+\alpha\|x\|_{1} .
$$

Since $f$ is convex and differentiable with gradient

$$
\nabla f(x)=A^{*}\left(I-P_{Q}\right) A x,
$$

$f_{\alpha}$ is convex, coercive, and subdifferentiable with the subdifferential

$$
\partial f_{\alpha}(x)=\partial f(x)+\alpha \partial\left(\|x\|_{1}\right) ;
$$

that is,

$$
\partial f_{\alpha}(x)=A^{*}\left(I-P_{Q}\right) A x+\alpha \partial\left(\|x\|_{1}\right) .
$$

By Corollary 2.3 and Lemma 2.4, the proof is finished. 
Theorem 3.3. Denote by $x_{\alpha}$ an arbitrary element of $S_{\alpha}$, then the following assertions hold:

(i) $\left\|x_{\alpha}\right\|_{1}$ is decreasing for $\alpha \in(0, \infty)$;

(ii) $\left\|\left(I-P_{Q}\right) A x_{\alpha}\right\|$ is increasing for $\alpha \in(0, \infty)$.

Proof. Let $\alpha>\beta>0$, for any $x_{\alpha} \in S_{\alpha}, x_{\beta} \in S_{\beta}$. We immediately obtain

$$
\begin{aligned}
& \frac{1}{2}\left\|\left(I-P_{Q}\right) A x_{\alpha}\right\|^{2}+\alpha\left\|x_{\alpha}\right\|_{1} \leq \frac{1}{2}\left\|\left(I-P_{Q}\right) A x_{\beta}\right\|^{2}+\alpha\left\|x_{\beta}\right\|_{1}, \\
& \frac{1}{2}\left\|\left(I-P_{Q}\right) A x_{\beta}\right\|^{2}+\beta\left\|x_{\beta}\right\|_{1} \leq \frac{1}{2}\left\|\left(I-P_{Q}\right) A x_{\alpha}\right\|^{2}+\beta\left\|x_{\alpha}\right\|_{1} .
\end{aligned}
$$

Adding up (3.13) and (3.14) yields

$$
\alpha\left\|x_{\alpha}\right\|_{1}+\beta\left\|x_{\beta}\right\|_{1} \leq \alpha\left\|x_{\beta}\right\|_{1}+\beta\left\|x_{\alpha}\right\|_{1},
$$

which implies $\left\|x_{\alpha}\right\|_{1} \leq\left\|x_{\beta}\right\|_{1}$. Hence (i) holds.

Using (3.14) again, we have

$$
\frac{1}{2}\left\|\left(I-P_{Q}\right) A x_{\beta}\right\|^{2} \leq \frac{1}{2}\left\|\left(I-P_{Q}\right) A x_{\alpha}\right\|^{2}+\beta\left(\left\|x_{\alpha}\right\|_{1}-\left\|x_{\beta}\right\|_{1}\right)
$$

which together with (i) implies

$$
\left\|\left(I-P_{Q}\right) A x_{\beta}\right\|^{2} \leq\left\|\left(I-P_{Q}\right) A x_{\alpha}\right\|^{2},
$$

and hence (ii) holds.

Let $\mathcal{F}=C \cap A^{-1}(Q)$, where $A^{-1}(Q)=\left\{x \in \mathbb{R}^{N}: A x \in Q\right\}$. In what follows, we assume that $\mathcal{F} \neq \emptyset$; that is, the solution set of SFP (1.1) is nonempty. The fact that $\mathcal{F}$ is nonempty closed convex set thus allows us to introduce the concept of minimum-norm solution of SFP (1.1) in the sense of norm $\|\cdot\|$ (induced by the inner product).

Definition 3.4 (see [14]). An element $x^{\dagger} \in \mathcal{F}$ is said to be the minimum-norm solution of SFP (1.1) in the sense of norm $\|\cdot\|$ if $\left\|x^{\dagger}\right\|=\inf _{x \in \mathcal{F}}\|x\|$. In other words, $x^{\dagger}$ is the projection of the origin onto the solution set $F$ of SFP (1.1). Thus there exists only one minimum-norm solution of SFP (1.1) in the sense of norm $\|\cdot\|$, which is always denoted by $x^{\dagger}$.

We can also give the concept of minimum-norm solution of SFP (1.1) in other senses.

Definition 3.5. An element $\tilde{x} \in \mathcal{F}$ is said to be a minimum-norm solution of SFP (1.1) in the sense of 1-norm if $\|\tilde{x}\|_{1}=\inf _{x \in \mathcal{F}}\|x\|_{1}$. We use $\mathcal{F}_{1}$ to stand for all minimum-norm solutions of SFP (1.1) in the sense of 1-norm and $\mathcal{F}_{1}$ is called the minimum-norm solution set of SFP (1.1) in the sense of 1-norm.

Obviously, $\mathcal{F}_{1}$ is a closed convex subset of $\mathscr{F}$. Moreover, it is easy to see that $\mp_{1} \neq \emptyset$. Indeed, taking a sequence $\left\{x_{n}\right\} \subset \mathcal{F}$ such that $\left\|x_{n}\right\|_{1} \rightarrow \inf _{x \in \mathcal{F}}\|x\|_{1}$ as $n \rightarrow \infty$, then $\left\{x_{n}\right\}$ 
is bounded. There exists a convergent subsequence $\left\{x_{n_{k}}\right\}$ of $\left\{x_{n}\right\}$. Set $\bar{x}=\lim _{k \rightarrow \infty} x_{n_{k}}$, then $\bar{x} \in \mathcal{F}$ since $\mathcal{F}$ is closed. On the other hand, using lower semicontinuity of the norm, we have

$$
\|\bar{x}\| \leq \lim _{k \rightarrow \infty}\left\|x_{n_{k}}\right\|=\inf _{x \in \mathcal{F}}\|x\|_{1}
$$

and this implies that $\bar{x} \in \mathscr{F}_{1}$.

However, $\mathcal{f}_{1}$ may contain more than one elements, in general (see Example 3.1, $\mp_{1}=$ $\{(x, y): x+y=1, x, y \geq 0\})$.

Theorem 3.6. Let $\alpha>0$ and $x_{\alpha} \in S_{\alpha}$. Then $\omega\left(x_{\alpha}\right) \subset \mathcal{F}_{1}$, where $\omega\left(x_{\alpha}\right)=\left\{x: \exists\left\{x_{\alpha_{k}}\right\} \subset\left\{x_{\alpha}\right\}, x_{\alpha_{k}} \rightarrow\right.$ $x$ weakly\}.

Proof. Taking $\tilde{x} \in \mathcal{F}_{1}$ arbitrarily, for any $\alpha \in(0, \infty)$, we always have

$$
\frac{1}{2}\left\|\left(I-P_{Q}\right) A x_{\alpha}\right\|^{2}+\alpha\left\|x_{\alpha}\right\|_{1} \leq \frac{1}{2}\left\|\left(I-P_{Q}\right) A \tilde{x}\right\|^{2}+\alpha\|\tilde{x}\|_{1} .
$$

Since $\tilde{x}$ is a solution of $\operatorname{SFP}(1.1),\left\|\left(I-P_{Q}\right) A \tilde{x}\right\|=0$. This implies that

$$
\frac{1}{2}\left\|\left(I-P_{Q}\right) A x_{\alpha}\right\|^{2}+\alpha\left\|x_{\alpha}\right\|_{1} \leq \alpha\|\tilde{x}\|_{1},
$$

then,

$$
\left\|x_{\alpha}\right\|_{1} \leq\|\tilde{x}\|_{1}
$$

thus $\left\{x_{\alpha}\right\}$ is bounded.

Take $\omega \in \omega\left(x_{\alpha}\right)$ arbitrarily, then there exists a sequence $\left\{\alpha_{n}\right\}$ such that $\alpha_{n} \rightarrow 0$ and $x_{\alpha_{n}} \rightarrow \omega$ as $n \rightarrow \infty$. Put $x_{\alpha_{n}}=x_{n}$. By Proposition 3.2, we deduce that there exists some $\xi_{n} \in$ $\partial\left(\left\|x_{n}\right\|_{1}\right)$ such that

$$
\left\langle A^{*}\left(I-P_{Q}\right) A x_{n}+\alpha_{n} \xi_{n}, \tilde{x}-x_{n}\right\rangle \geq 0 .
$$

This implies that

$$
\left\langle\left(I-P_{Q}\right) A x_{n}, A\left(\tilde{x}-x_{n}\right)\right\rangle \geq \alpha_{n}\left\langle\xi_{n}, x_{n}-\tilde{x}\right\rangle .
$$

Since $A \tilde{x} \in Q$, the characterizing inequality (2.2) gives

$$
\left\langle\left(I-P_{Q}\right) A x_{n}, A \tilde{x}-P_{Q}\left(A x_{n}\right)\right\rangle \leq 0,
$$

then,

$$
\left\|\left(I-P_{Q}\right) A x_{n}\right\|^{2} \leq\left\langle\left(I-P_{Q}\right) A x_{n}, A\left(x_{n}-\tilde{x}\right)\right\rangle .
$$


Combining (3.23) and (3.25), we have

$$
\begin{aligned}
\left\|\left(I-P_{Q}\right) A x_{n}\right\|^{2} & \leq \alpha_{n}\left\langle\xi_{n}, \tilde{x}-x_{n}\right\rangle \\
& \leq \alpha_{n}\left\|\xi_{n}\right\|_{\infty}\left\|\tilde{x}-x_{n}\right\|_{1} \\
& \leq 2 \alpha_{n}\|\tilde{x}\|_{1} .
\end{aligned}
$$

Consequently, we get

$$
\lim _{n \rightarrow \infty}\left\|\left(I-P_{Q}\right) A x_{n}\right\|=0
$$

Furthermore, noting the fact that $x_{n} \rightarrow \omega$ and $I-P_{Q}$ and $A$ are all continuous operators, we have $\left(I-P_{Q}\right) A \omega=0$; that is, $A \omega \in Q$; thus, $\omega \in \mathcal{F}$. Since $\tilde{x}$ is a minimum-norm solution of SFP (1.1) in the sense of 1-norm, using (3.21) again, we get

$$
\|\omega\|_{1} \leq \liminf _{n \rightarrow \infty}\left\|x_{n}\right\|_{1} \leq\|\tilde{x}\|_{1}=\min \left\{\|x\|_{1}: x \in \mathcal{F}\right\}
$$

Thus we can assert that $\omega \in \mathscr{F}_{1}$ and this completes the proof.

Corollary 3.7. If $\mathcal{F}_{1}$ contains only one element $\tilde{x}$, then $x_{\alpha} \rightarrow \tilde{x},(\alpha \rightarrow 0)$.

Remark 3.8. It is worth noting that the minimum-norm solution of SFP (1.1) in the sense of norm $\|\cdot\|$ is very different from the minimum-norm solution of SFP (1.1) in the sense of 1-norm. In fact, $x^{\dagger}$ may not belong to $\mp_{1}$ ! The following simple example shows this fact.

Example 3.9. Let $C=\{(x, y): x+2 y \geq 2, x \geq 0, y \geq 0\}, Q=\{(x, y): x+y=1, x \geq 0, y \geq 0\}$, and

$$
A=\left(\begin{array}{ll}
\frac{1}{2} & 0 \\
0 & 1
\end{array}\right) .
$$

It is not hard to see that $A: \mathbb{R}^{2} \rightarrow \mathbb{R}^{2}$ is a bounded linear operator and $A(x, y)^{T}=((1 / 2) x$, $y)^{T}$, for all $(x, y) \in C$. Obviously, $\mathcal{F}=\{(x, y): x+2 y=2, x \geq 0, y \geq 0\}, x^{\dagger}=(2 / 5,4 / 5)$, but $\mathcal{F}_{1}=\{(0,1)\}$. Hence, $x^{\dagger} \in \mathcal{F} \backslash \mathcal{F}_{1}$.

\section{Acknowledgments}

This work was supported by the Fundamental Research Funds for the Central Universities (ZXH2012K001) and in part by the Foundation of Tianjin Key Lab for Advanced Signal Processing. W. Zhu was also supported by the Postgraduate Science and Technology Innovation Funds (YJSCX12-22).

\section{References}

[1] Y. Censor and T. Elfving, "A multiprojection algorithm using Bregman projections in a product space," Numerical Algorithms, vol. 8, no. 2-4, pp. 221-239, 1994. 
[2] Y. Yao, R. Chen, G. Marino, and Y. C. Liou, "Applications of fixed point and optimization methods to the multiple-sets split feasibility problem," Journal of Applied Mathematics, vol. 2012, Article ID 927530 , 21 pages, 2012.

[3] C. Byrne, "Iterative oblique projection onto convex sets and the split feasibility problem," Inverse Problems, vol. 18, no. 2, pp. 441-453, 2002.

[4] C. Byrne, "A unified treatment of some iterative algorithms in signal processing and image reconstruction," Inverse Problems, vol. 20, no. 1, pp. 103-120, 2004.

[5] B. Qu and N. Xiu, "A note on the CQ algorithm for the split feasibility problem," Inverse Problems, vol. 21, no. 5, pp. 1655-1665, 2005.

[6] H. K. Xu, "A variable Krasnosel'skii-Mann algorithm and the multiple-set split feasibility problem," Inverse Problems, vol. 22, no. 6, pp. 2021-2034, 2006.

[7] Q. Yang, "The relaxed CQ algorithm solving the split feasibility problem," Inverse Problems, vol. 20, no. 4, pp. 1261-1266, 2004.

[8] Q. Yang and J. Zhao, "Generalized KM theorems and their applications," Inverse Problems, vol. 22, no. 3, pp. 833-844, 2006.

[9] Y. Yao, J. Wu, and Y. C. Liou, "Regularized methods for the split feasibility problem," Abstract and Applied Analysis, vol. 2012, Article ID 140679, 15 pages, 2012.

[10] X. Yu, N. Shahzad, and Y. Yao, "Implicit and explicit algorithms for solving the split feasibility problem ," Optimization Letters. In press.

[11] F. Wang and H. K. Xu, "Cyclic algorithms for split feasibility problems in Hilbert spaces," Nonlinear Analysis, Theory, Methods and Applications, vol. 74, no. 12, pp. 4105-4111, 2011.

[12] H. K. Xu, "Averaged mappings and the gradient-projection algorithm," Journal of Optimization Theory and Applications, vol. 150, no. 2, pp. 360-378, 2011.

[13] H. K. Xu, "Iterative methods for the split feasibility problem in infinite-dimensional Hilbert spaces," Inverse Problems, vol. 26, no. 10, Article ID 105018, 2010.

[14] H. K. Xu and F. Wang, "Approximating curve and strong convergence of the CQ algorithm for the split feasibility problem," Journal of Inequalities and Applications, vol. 2010, Article ID 102085, 13 pages, 2010.

[15] M. R. Kunz, J. H. Kalivas, and E. Andries, "Model updating for spectral calibration maintenance and transfer using 1-norm variants of tikhonov regularization," Analytical Chemistry, vol. 82, no. 9, pp. 3642-3649, 2010.

[16] X. Nan, N. Wang, P. Gong, C. Zhang, Y. Chen, and D. Wilkins, "Gene selection using 1-norm regulariza-tion for multi-class microarray data," in Proceedings of the IEEE International Conference on Bioinformatics and Biomedicine (BIBM '10), pp. 520-524, December 2010.

[17] X. Nan, Y. Chen, D. Wilkins, and X. Dang, "Learning to rank using 1-norm regularization and convex hull reduction," in Proceedings of the 48th Annual Southeast Regional Conference (ACMSE '10), Oxford, Miss, USA, April 2010.

[18] H. W. Park, M. W. Park, B. K. Ahn, and H. S. Lee, "1-Norm-based regularization scheme for system identification of structures with discontinuous system parameters," International Journal for Numerical Methods in Engineering, vol. 69, no. 3, pp. 504-523, 2007.

[19] J. P. Aubin, Optima and Equilibria: An Introduction to Nonliear Analysis, vol. 140 of Graduate Texts in Mathematics, Springer, Berlin, Germany, 1993.

[20] H. W. Engl, M. Hanke, and A. Neubauer, Regularization of Inverse Problems, vol. 375 of Mathematics and Its Applications, Kluwer Academic, Dordrecht, The Netherlands, 1996. 


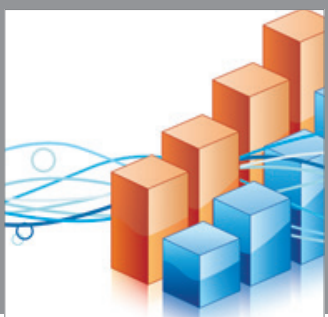

Advances in

Operations Research

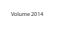

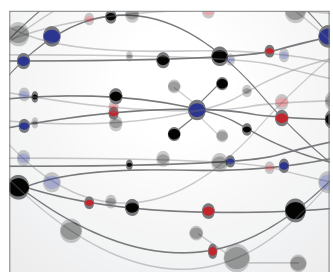

\section{The Scientific} World Journal
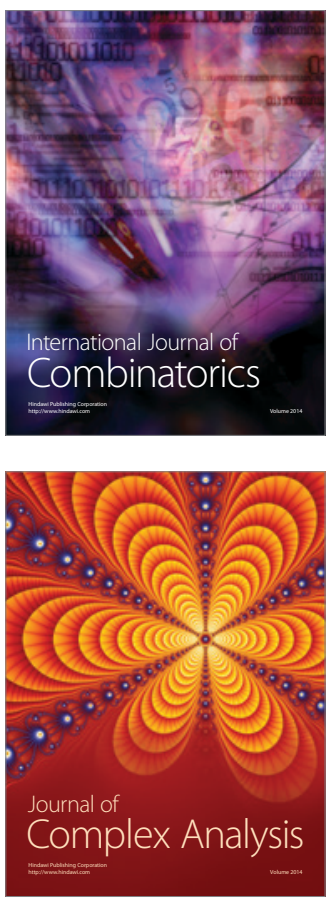

International Journal of

Mathematics and

Mathematical

Sciences
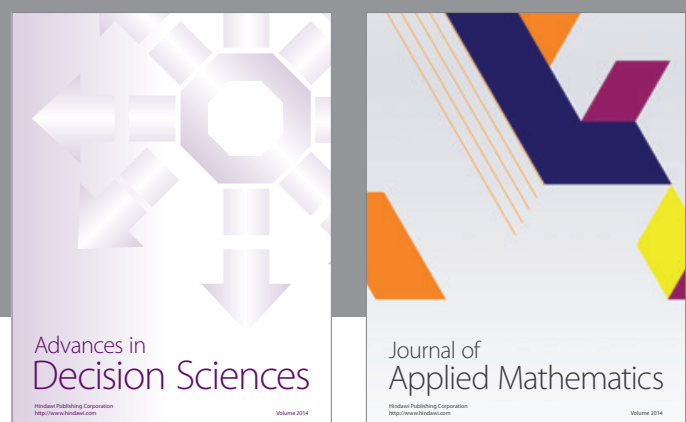

Journal of

Applied Mathematics
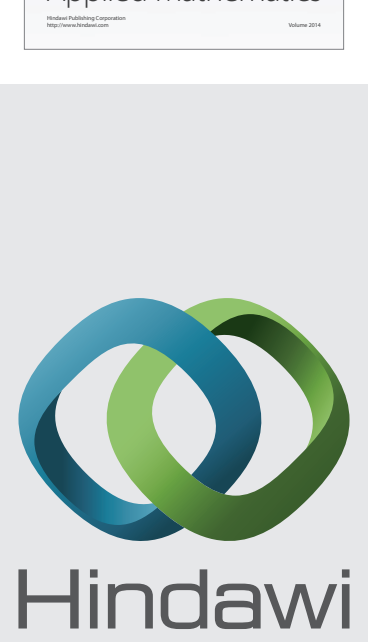

Submit your manuscripts at http://www.hindawi.com
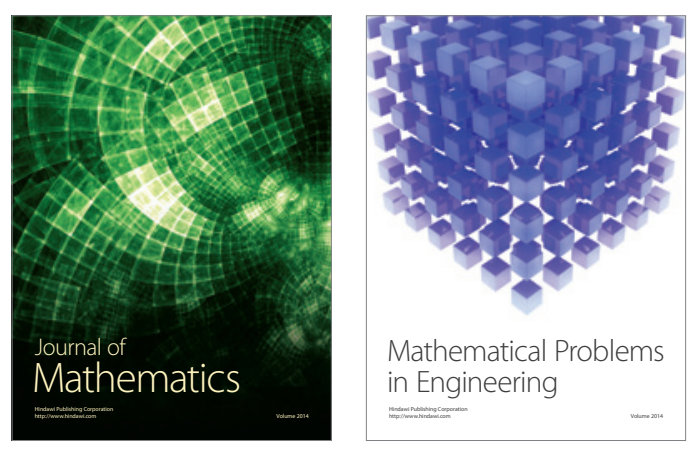

Mathematical Problems in Engineering
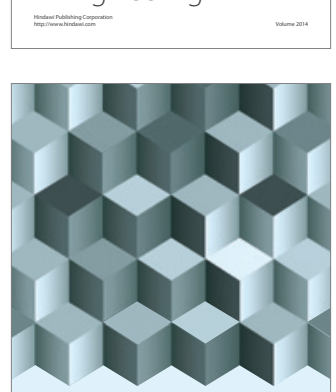

Journal of

Function Spaces
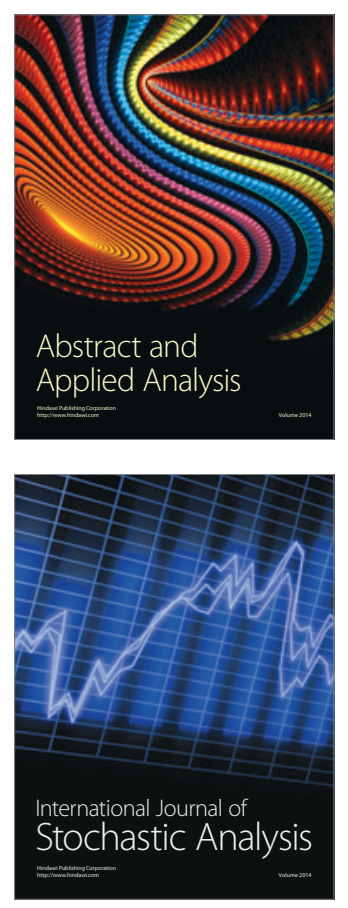

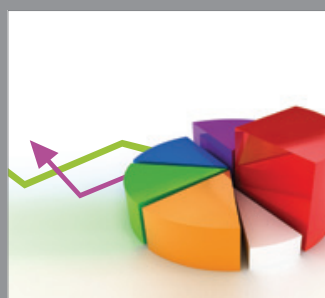

ournal of

Probability and Statistics

Promensencen
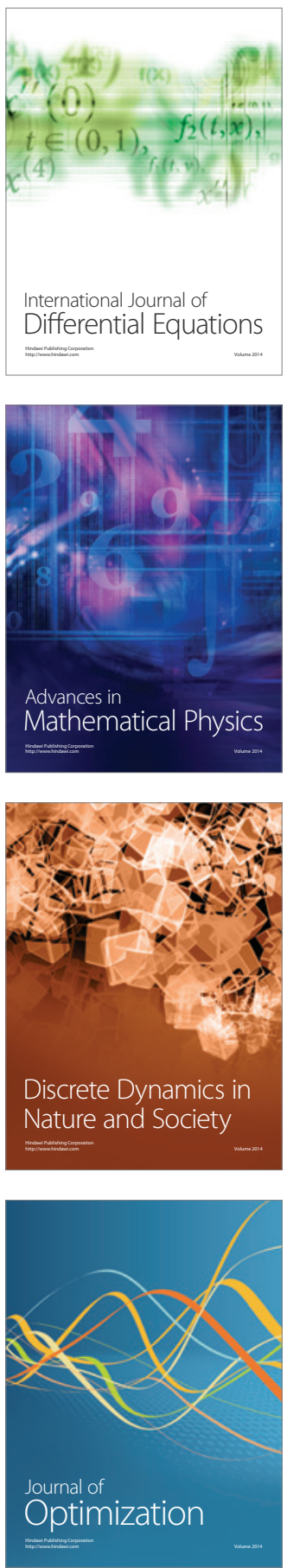